\title{
Automatic solution for detection, identification and biomedical monitoring of a cow using remote sensing for optimised treatment of cattle
}

\author{
Yevgeny Beiderman, ${ }^{1}$ Mark Kunin,, Eli Kolberg, ${ }^{2}$ Ilan Halachmi, ${ }^{1}$ Binyamin Abramov, ${ }^{2}$ \\ Rafael Amsalem, ${ }^{2}$ Zeev Zalevsky ${ }^{2}$ \\ 'Institute of Agricultural Engineering, Agricultural Research Organisation (ARO), the \\ Volcani Center, Bet-Dagan; ${ }^{2}$ Faculty of Engineering, Bar-Ilan University, Ramat-Gan, Israel
}

\begin{abstract}
In this paper we show how a novel photonic remote sensing system assembled on a robotic platform can extract vital biomedical parameters from cattle including their heart beating, breathing and chewing activity. The sensor is based upon a camera and a laser using selfinterference phenomena. The whole system intends to provide an automatic solution for detection, identification and biomedical monitoring of a cow. The detection algorithm is based upon image processing involving probability map construction. The identification algorithms involve well known image pattern recognition techniques. The sensor is used on top of an automated robotic platform in order to support animal decision making. Field tests and computer simulated results are presented.
\end{abstract}

\section{Introduction}

Automatic robotic cattle management is an appealing problem which is of an interest for the agricultural and dairy community. A detection, identification and physical biomedical check of a cow is usually done by different sensors per each task. Previously, several attempts were made to monitor the cows' positioning. An image processing technique based on a spatial segmentation of cows produced from images filmed from top-mounted cameras was applied (Alhanati et al., 2010). However, this technique suffers from a dependency on proper illumination and cows' surface texture. Radio-frequency identification (RFID) technique was introduced to monitor a location of

Correspondence: Eli Kolberg, Faculty of Engineering, Bar-Ilan University, Ramat-Gan 52900, Israel.

E-mail: eli.kolberg@biu.ac.il

Key words: speckles, remote sensing, cattle identification, search methods.

Received for publication: 5 July 2014.

Accepted for publication: 6 October 2014.

(C) Copyright Y. Beiderman et al., 2014

Licensee PAGEPress, Italy

Journal of Agricultural Engineering 2014; XLV:418

doi:10.4081/jae.2014.418

This article is distributed under the terms of the Creative Commons Attribution Noncommercial License (by-nc 3.0) which permits any noncommercial use, distribution, and reproduction in any medium, provided the original author(s) and source are credited. cows, however it requires a specific detector location (Stankovsky et al., 2012), i.e. it can detect a location in a predefined area. Kim and Young Jung (2013) introduced recently a combined technique of RGB space filtering and RFID monitoring.

Biomedical monitoring of bovine animals can help better maintain welfare of the animals and therefore help in establishing a profitable business at an agricultural farm. For instance, the heart rate and respiration rate measurements indicate energy balance in ruminants (Brosh, 2007) and advance early detection of an outbreak diseases and animal stress (Brosh et al., 2006).

In this paper we introduce a combined sensor for detection, identification and extraction of bio-medical parameters off a cow. Our proposal is based on a completely different approach which comprises a laser projection and camera filming of a back-reflected laser light. This system is capable to distinguish each cow independently and to monitor remotely $r$, various vital biomedical parameters such as breathing, heart beat rate, and chewing activity. The proposed technique is not affected by the illumination intensity fluctuations, the contrast changes and the cows' skin texture. The laser is used to produce biomedical information off a cow. The camera has numerous usages: first, to grab the reflected laser information, second, to identify the cows' individual freeze branding number and finally to navigate the system in the barn space.

Our laser illuminates a cow with a collimated laser beam. The reflected light is grabbed by the camera, while the laser self-interference phenomena (a speckle pattern) are imaged. The speckles are self-interference random patterns (Dainty, 1989) and have the remarkable quality that each individual speckle serves as a reference point from which one may track the changes in the phase of the light that is being scattered from the surface (Dainty, 1989).

Because of that, speckle techniques such as electronic speckle-pattern interferometry have been widely used for displacement measuring and vibration analysis (amplitudes, slopes and modes of vibration) as well as characterisation of deformations (Leedertz, 1970; Pedersen, 1982; Peters and Ranson, 1982; Rastogi and Jacquot, 1987; Takai et al., 1980; Chu et al., 1985; Uno et al., 1996). In case of an object deformations measurement, one subtracts the speckle pattern before the deformation has occurred (due to change in loading, change in temperature, etc.) from the pattern after deformation has occurred. This procedure produces correlation fringes that correspond to the object's local surface displacements between the two exposures. From the fringe pattern both the magnitude and the direction of the object's local surface displacement is determined (Leedertz, 1970; Rastogi and Jacquot, 1987). Usage of speckles was also applied for improving the resolving capabilities of imaging sensors (Garcia et al., 2008) as well as ranging and 3D estimation (Garcia et al., 2005).

In our sensing configuration, the detection is obtained via fast imaging camera that observes the temporal intensity fluctuations of the imaged speckles pattern and their trajectory. In order to allow cor- 
relating the trajectory with the movement of the speckle patterns we had to properly defocus our imaging lens. We propose not to focus the camera on the object, instead to focus on a far or close field such that the object itself is defocused. Doing that makes the movement of the object (its vibrations) to cause a lateral shift of the speckles pattern. Actually due to this defocusing, the movement of the object instead of constantly changing the speckle pattern creates a situation in which we see the same speckle pattern moving or vibrating in the transversal plane. This feature allows tracking the maxima intensity spots, the extraction of the trajectory movement. The extraction of the skin surface movement with nanometric accuracy (Zalevsky and Garcia, 2007; Beiderman et al., 2011a) can produce an estimation of the blood viscosity as well as its pulsation. Based on the skin movement, one may extract important biomedical parameters from a distance, without a contact, in a non-invasive way. In previous works we were able to develop photonic sensor in order to measure human biomedical parameters (Zalevsky et al., 2009; Beiderman et al., 2010; Beiderman et al., 2011b; Cojoc et al., 2012; Beiderman et al., 2012; Shenhav et al., 2012; Margalit et al., 2014). Our previous measurements included heartbeat rate and shape (Zalevsky et al., 2009), blood pulse pressure (Beiderman et al., 2010), estimation for glucose concentration in the blood stream (Beiderman et al., 2011b), intra-ocular pressure (IOP) in the eye (Margalit et al., 2014), estimation of amount of alcohol in the blood stream (Shenhav et al., 2012) and even using our sensor as a tool for early detection of malaria infected red blood cells (Cojoc et al., 2012). For some of those parameters such glucose concentration and IOP the alternative is invasive or at least non pleasant procedure for the human patient. Note that the proposed system can be realised in a very simple and low price manner by simply using common components of a conventional optical mouse (Beiderman et al., 2012). However all the above studies followed by developing human applications and were tested in lab conditions. Working with farm animal introduces further challenge - the existence of pelt - animal skin with thick fat layer (Halachmi et al., 2008) and non-cooperative nature of the animals to the measurement procedures. Therefore, field tests were performed.

The whole optical system will be assembled on an autonomous mobile robot. In order to navigate and identify the robotic platform uses a special navigation algorithm along with several image processing algorithms that were developed. Our cow detection algorithm is based upon probabilities map construction. The map is transformed into an approximation of sum of 2D Gaussian curves, where each one is statistically independent of others and with similar variance between axes (XY). The algorithm is compared to well-known navigation techniques, like the greedy probabilistic algorithm (Pelikan et al., 2002).

Cow identification is based on recognition of individual numbers (freeze branding) that are branded in each cow. Freeze branding (Battaglia, 2001) serves as a method of animal identification from a larger distance than is feasible with ear tags. Freeze branding, similar to hot branding, involves the use of branding irons, with letters and numbers, being chilled in liquid nitrogen or dry ice and alcohol. Freeze brands are typically 4 -inch high numbers and letters; they should be placed in the shoulder or buttocks area. In this paper we deal with freeze brands of three-digit numbers, which are located on the upper cow's buttocks area. Each cow is marked with 3-digits number surrounded by a white rectangle (similar to regular car license number). For number recognition we use the well-known algorithm described in (Shapiro et al., 2004).

In this paper we show a construction of an automated photonic sensor placed on top of a robotic platform used in cowsheds or barns, while the purpose of our experiments was to automatically estimate the biomedical state of the cows. Special algorithms were implemented in a software package in order to operate a robotic system equipped with the sensor. First the system detects and identifies a cow in cattle locat- ed in a barn, while later captures the bio-medical parameters of that cow. The system operates constantly with no interference to the flock. The data of the cow number along with its biomedical information can be sent to a remote computer. The software algorithm may signal that one or more cows need to be treated. A person in charge can look at the data presented in the computer and take the necessary action. That way, it saves time and costs of human inspection and alert. In this paper we will discuss the sensor and camera system alone.

\section{Theoretical explanation}

\section{Biomedical sensor}

In general, the system is based on a temporal tracking of secondary speckle patterns. The laser illuminates the region of interest where the measurement should be performed. Speckle patterns are generated on the detector of the camera after light is being back reflected from the illuminated surface. By properly adjusting the optics of the camera it was shown that tilting the inspected back reflecting surface generates movement rather than change in the speckle pattern distribution. Therefore, it is possible to track the temporal shifts and movements of this random pattern by performing proper correlation operations (Leedertz, 1970). The applied tracking allows determining both the magnitude and the direction of the subject's local surface displacement (Rastogi and Jacquot, 1987) when applying the exact image processing correlation-based algorithm that is realised via MATLAB and C++ software codes. If the back reflecting skin is close to main blood arteries then the remotely measured temporal movements can be translated into estimation of various vital biomedical parameters such as heart beating. Breathing activity of cows can be recorded from the central part of a body, while the chewing activity is the best monitored near the jaws.

\section{Software algorithm development}

The software algorithm research comes to help the robotic system to navigate and identify a cow in order to operate the optical sensor. The cow identification process includes two components: the search algorithm and the cow number identification. The system that integrates the optical sensor with a digital camera can be placed on a moving robotic system above a cowshed area. When the optical sensor identifies a cow, the system stops, collects the cow data and number and transmits it to a host computer. This process is in the design stage.

\section{Search algorithm}

The camera is hanged $3 \mathrm{~m}$ above the ground and can move autonomously along tracks that cover all of the cowshed area. The optical sensor is attached to the camera. The snail path algorithm is used for area covering. The area will be divided into cells of $50 \times 50 \mathrm{~cm}$ each. First time the camera with the sensor will scan the whole area and assign probability of cow locations in each cell. This probabilities map is transformed into an approximation of sum of 2D Gaussian curves, where each one is statistically independent of others and with similar variance between axes (XY). This map is stored dynamically for analysis purpose. The probability function can be regarded as a topology where $\mathrm{XY}$ axes are cell locations and $\mathrm{Z}$ axis is the probability of cow presence. Each 2D Gaussian is defined as presented in Equation 1.

$$
f(x, y)=\frac{1}{2 \pi \sigma_{x} \sigma_{y}} e^{-\left[\left(x-\mu_{x}\right)^{2} /\left(2 \sigma_{x}^{2}\right)+\left(y-\mu_{y}\right)^{2} /\left(2 \sigma_{y}^{2}\right)\right]}
$$


A cell weight is the probability of cow presence and a path weight is the product of the probabilities of cow presence of each cell in the path. The path starts from the current camera position going to the location with the highest probability. The camera scans the area all the way to this location. In any point, when the sensor detects a cow, the camera stops moving, takes a picture, and performs image processing for cow identification. The cow number along with the sensor data is sent to a remote computer. We will discuss the image processing phase later. Then the camera continues scanning the area.

The camera stops scanning a local area when the probabilities of cow presence are below a predefined threshold or lower than those in other area. When that happens, the camera moves to the next highest probability rank area. The scanning process continues to all areas in descending order of probabilities. The scanning cycle restarts when all cells are scanned or all cows are found in the current cycle.

We will define efficiency as appears in Equation 2.

$$
\text { efficiency }=\sum_{j \in \text { seps }} \frac{P_{j} \frac{1}{t_{j}}}{T} \quad 0<t_{j}<T, 0<P_{j}<1
$$

where:

$T$ is the overall scan time;

$t_{j}$ is the time of arrival to cell $\mathrm{j}$;

$P_{j}$ is a priory probability of $\mathrm{j}$ cell.

We compared the algorithm to snake search and greedy probabilistic algorithm. The algorithm we introduce is presented with best and worth probability threshold for determining when to leave a local area towards the next point of interest.

\section{Cow identification}

Cow identification is based on recognition of individual numbers (freeze branding) that are branded in each cow. Freeze brands (Battaglia, 2001) are typically 4-inch high numbers and letters; in this paper we deal with freeze brands of three-digit numbers, which are located on the upper cow's buttocks area. Each cow is marked with 3digits number surrounded by a white rectangle (similar to regular car license number). A permanent brand as presented in Figure 1.

In determining the exact location of the cow, we support two scenarios: i) each cow has permanent stall with known location; ii) cows do not have a permanent place.

It should be capable of: i) working indoor and outdoor; ii) working in a wide range of illumination conditions; iii) being invariant to size, scale and stroke thickness; iv) being robust to camera-cow relative movement.

The most popular approach is based on edge detection, gradient and other variants of intensity derivatives. These techniques are sensitive to noise and illumination variation, therefore they need to be supported or complemented by other methods.

We use the well-known adaptive image extraction algorithm, which works with single frame grey-level images, obtained at different daytime and weather conditions, as an input. The main stages of this algorithm are shown in Figure 2, and widely described in Shapiro et al. (2004).

\section{Experimental setup}

Our experimental sensor was tested in a field, while the robotic platform algorithms were simulated by a computer. In the future, the robotic platform will be equipped with the biomedical sensor and operated by

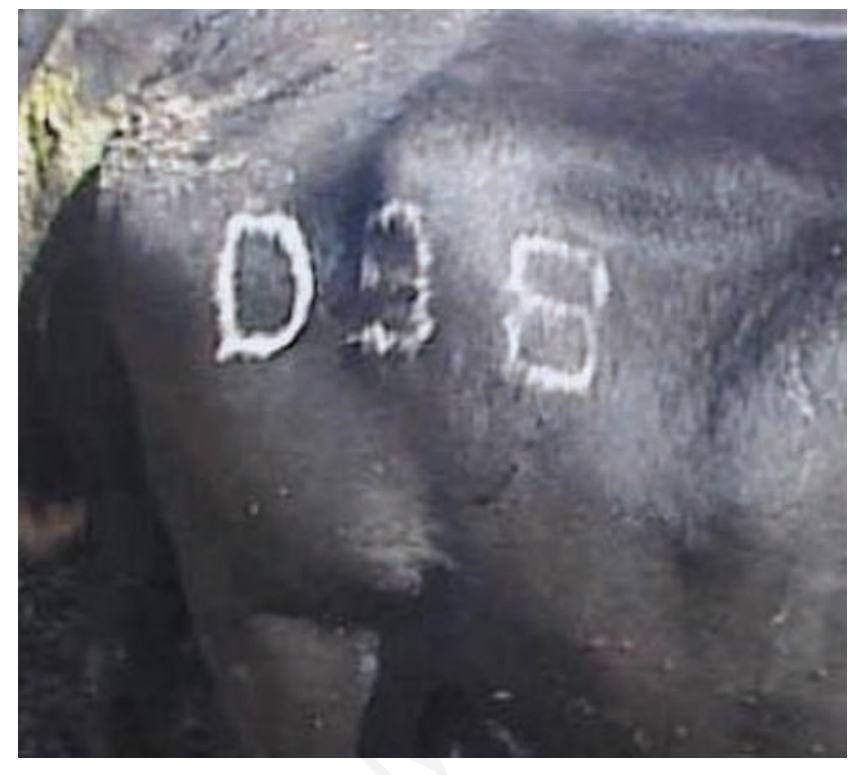

Figure 1. Freeze brand number D98 on a cow.

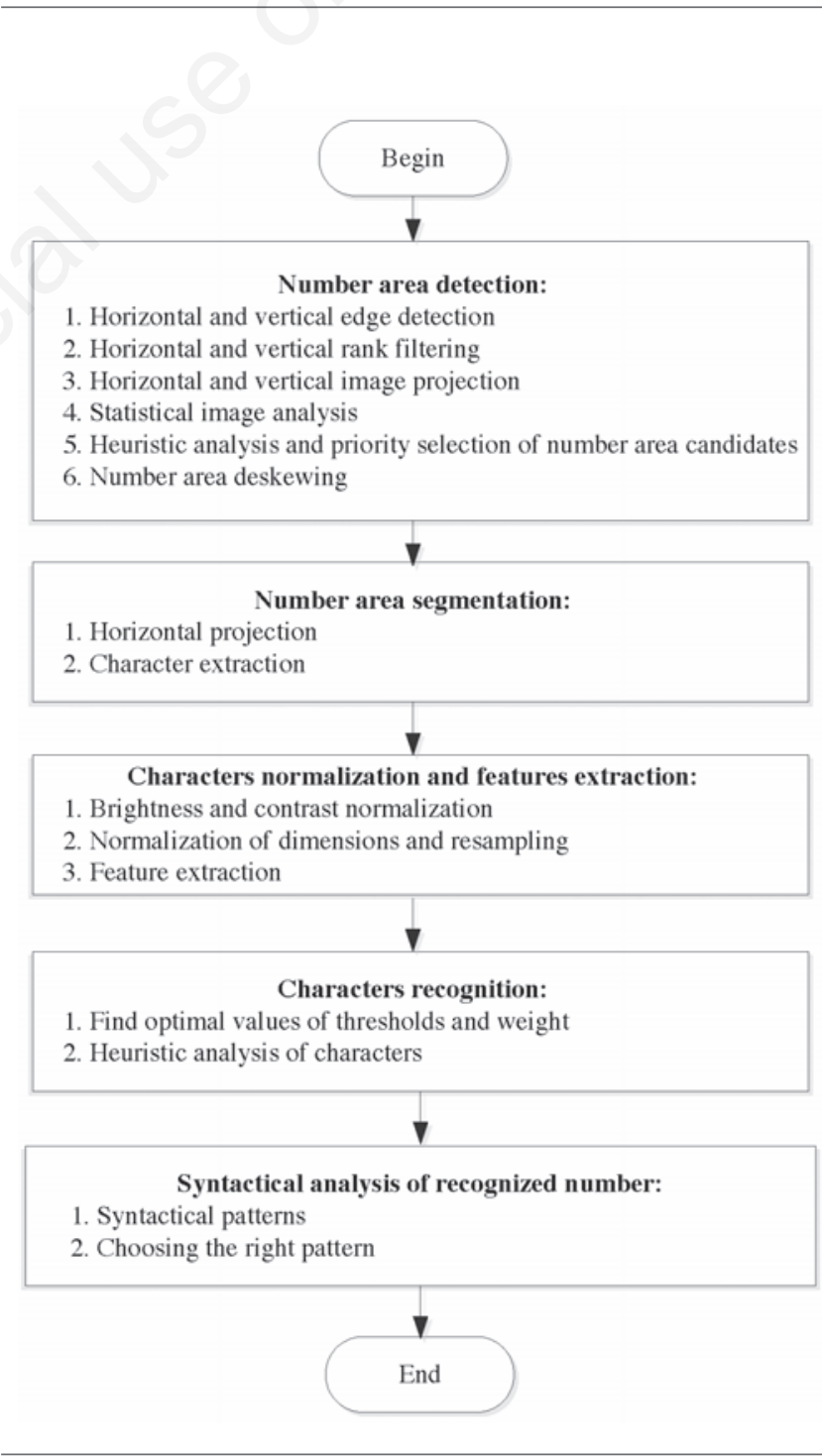

Figure 2. Number recognition algorithm flow chart. 
the proposed algorithms. We tested two cases using our optical sensor: i) the cow was located at a distance of approximately $30 \mathrm{~cm}$ from the optical sensing device. A laser beam was pointing to the neck of the cow. The gold-reference was a Polar watch with a belt strap sensor well tied to the animal behind the forth legs, oiled with a conductivity gel (Brosh, 2007; Brosh et al., 2006); ii) the cow was located at a distance of approximately $2 \mathrm{~m}$ from the optical sensing device. A laser beam was pointing to the neck and to the stomach of the cow (to detect its breathing activity) using the same gold reference.

Two cows were checked at each time; total of 6 cows were checked (total of 3 field test). In Figure 3 one can see the constrained sensor module. The module is assembled on a tripod equipped with a camera (PixelLink B741) and the laser (Photop D2100). The camera is connected to the computer (Dell M4600), which process the acquired video information using Matlab software. In Figure 4 one may see the images of the experimental scenario and configuration installed at the farm located in Newe Yaar, the Agricultural Research Organisation (AR0)'s northern research centre, Israel.

\section{Experimental processing and results}

As previously mentioned three biomedical parameters of the cattle were measured in a remote manner.

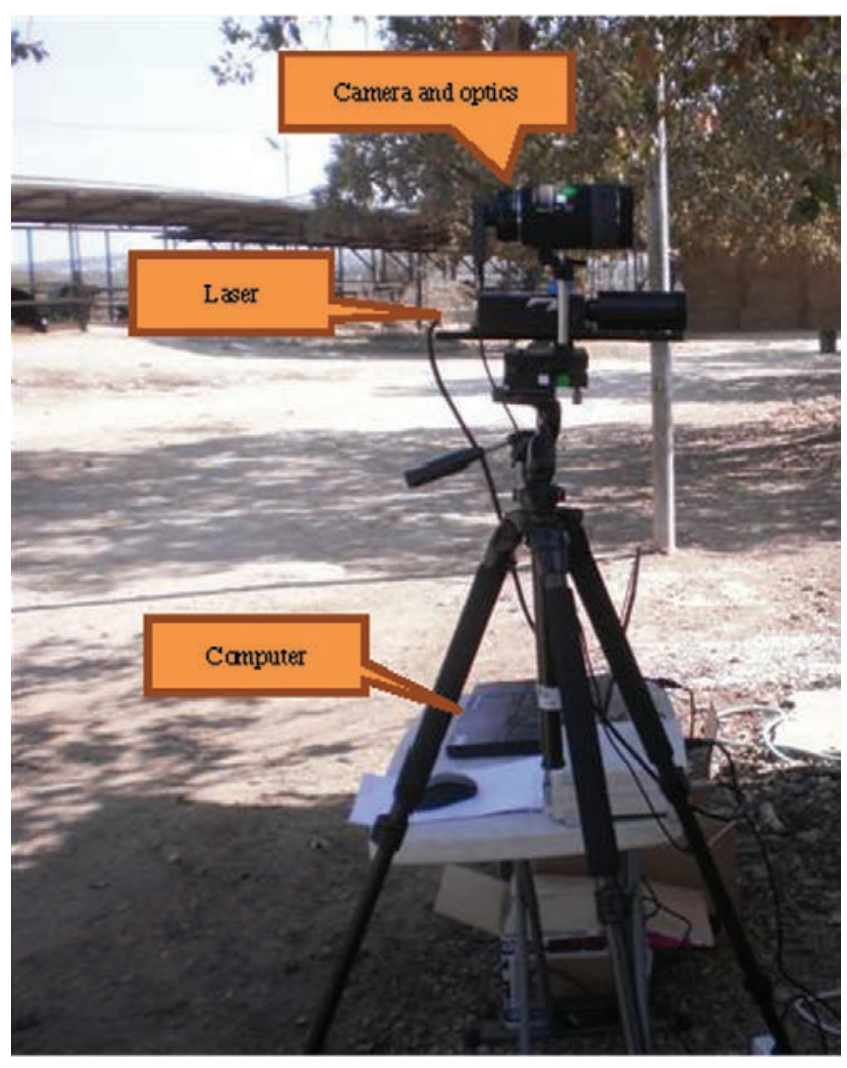

Figure 3. The experimental optical sensing device used on site. The device consists of a camera and a laser built on a tripod. The laser projects a beam on the cow while images taken by the camera are processed by a connected computer.
The system comprises of a laser and a camera with a capability to work at high frame rate. The rate depends on the application, in our case a frame rate of $500-1000 \mathrm{~Hz}$ over a small region of interest is enough. In general, the system is based on a temporal tracking of secondary speckle patterns. The laser illuminates the region of interest where the measurement should be performed. Speckle patterns are generated on the detector of the camera after light is being back reflected from the illuminated surface as shown in Figure 5. By properly adjusting the optics of the camera it was shown that tilting of the inspected back reflecting surface generates movement rather than change in the speckle pattern distribution. Therefore, it is possible to track the temporal shifts and movements of this random pattern by performing proper correlation operations (Leedertz, 1970). The applied tracking allows determining both the magnitude and the direction of the subject's local surface displacement (Rastogi and Jacquot, 1987) when applying the exact image processing correlation-based algorithm that is realised via MATLAB and $\mathrm{C}++$ software codes. If the back reflecting skin is close to main blood arteries then the remotely measured temporal movements can be translated into estimation of various vital biomedical parameters such as heart beating. Breathing and chewing activity of cows can be recorded from the central body part, while the chewing is best to be monitored near the jaw area. The automatic recognition of the peaks in the detected signals (the heart beat signature or a breathing signature) was done using Matlab software function findpeaks.

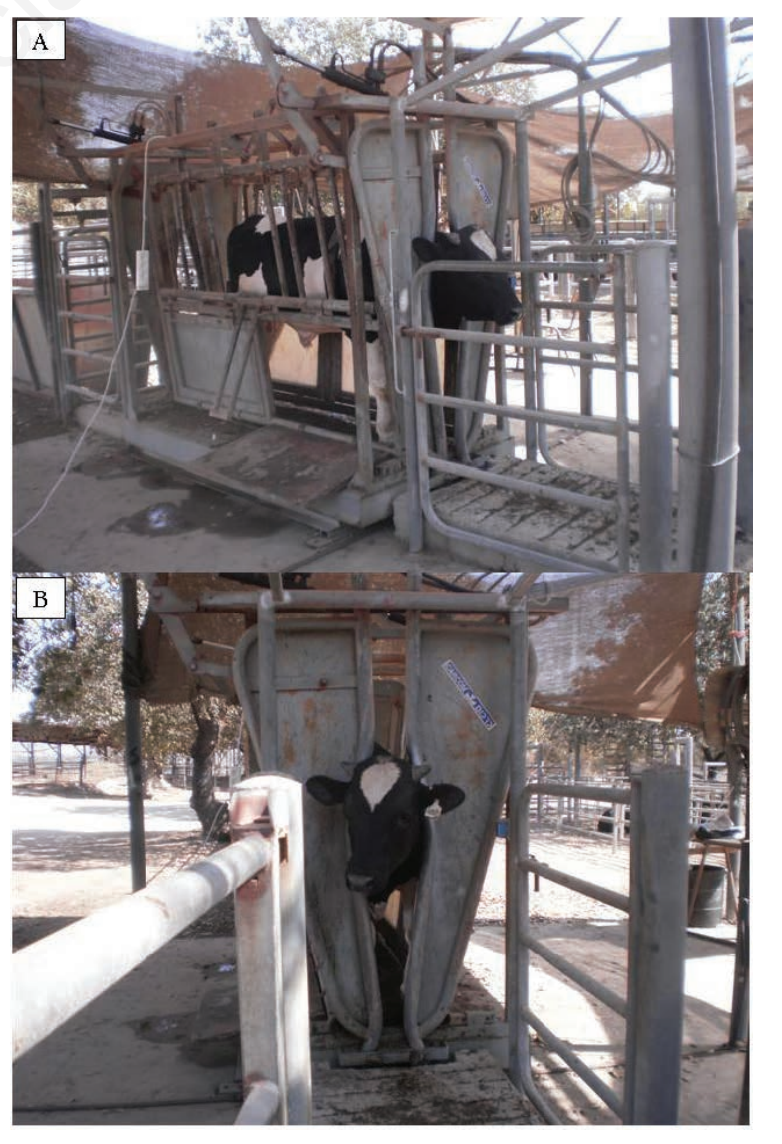

Figure 4. Cows used in the experiments. A and B) The cows were locked in a special cage while their movements were limited. 


\section{Breathing measurement}

We found a very clear periodic signal with a breath shape of the cows. The reference measurement (manual) matched our measurements. Example of the reconstructed signal is shown in Figure 6A. In that specific measurement our sensor recovered breathing rate of 60 times per minute. The reference of the breathing measurement was a manual stethoscope score. The reference count was 59 times per minute.

\section{Pulse measurement}

We measured the heart beating pulse and found a very clear periodic signal with a very determined pulse shape. The reference measurement (made with Polar watch) matches our measurements. Example of the reconstructed signal is seen in Figure $6 \mathrm{~B}$. There, the measured heart beating rate was 72 beats per minute. Exactly the same measurement was also obtained with our reference measurement device (Polar watch).

\section{Chewing activity}

When measuring the chewing activity of the cow with our photonic sensor we, in this case as well, found a very clear periodic signal. The reference counting matches our measurements. An example of the reconstructed signal is shown in Figure 6C. The vertical axis refers to amplitude measured in pixels. The measurement produced by our sensor gave the value of 60 (episode/min) while the reference measurement produced the same readout as well.

\section{Statistics}

We have collected dozens of heart beats measurements from each cow. In Figure 7A and B we show a collection of two of the cows' measurements. The correlation coefficient for the two of the collection (Figure 5A and B) are 0.967 and 0.982 respectively. The reference of the heart beat measurement was Polar watch as mentioned before. Figure 7C shows our breathing measurement on one of the cows, while the

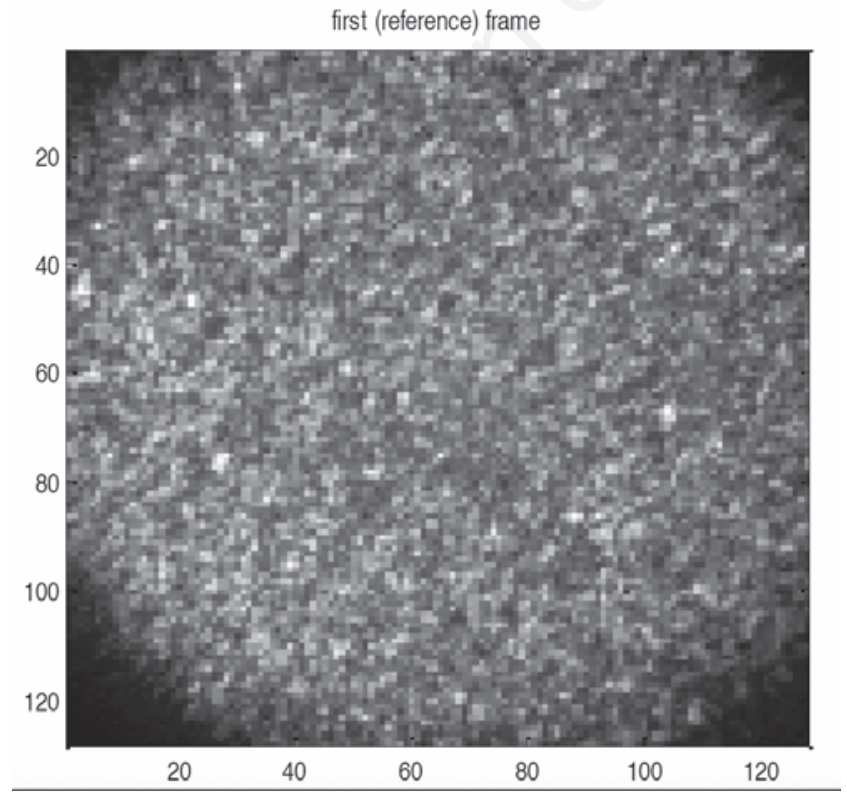

Figure 5. Image of a speckle patter as it is collected by a camera.
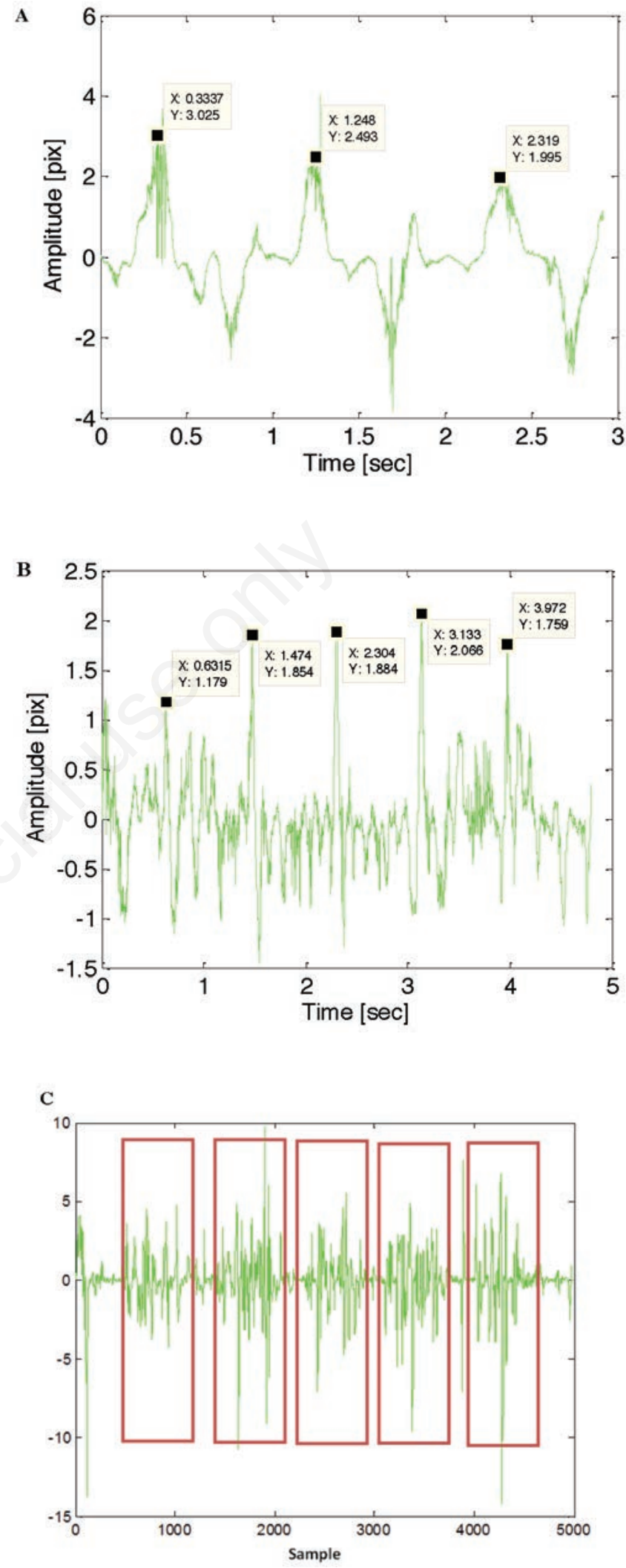

Figure 6. Cows' measurement output: A) Breath measurements. Each breath peak is marked by black square with $(\mathrm{X}, \mathrm{Y})$ position; B) Pulse measurements' raw signal. Each pulse peak is marked by black square with $(\mathrm{X}, \mathrm{Y})$ position; C) Chewing of a cow. Each chewing episode is enclosed in a red rectangle. Sampling rate of the camera $=1000 \mathrm{~Hz}$. 
correlation coefficient was 0.9946 in that case. The reference of the breathing measurement was a manual stethoscope score as mentioned before. The blue dashed line denotes to the reference measurement, while the red solid line to the optical readout.

\section{Software algorithm simulation: search algorithm}

Figure 8 presents a comparison of the algorithm to snake search and greedy probabilistic algorithm. The algorithm is presented with best and worth probability threshold for determining when to leave a local area towards the next point of interest.

We further studied the optimal threshold values as a function of the peak numbers in the area. Since the map is built on the first run, it is possible to calculate in advance the suitable threshold value that will produce the best results for a specific case.

Figure 9 presents data received from 605 runs with 5 different maps
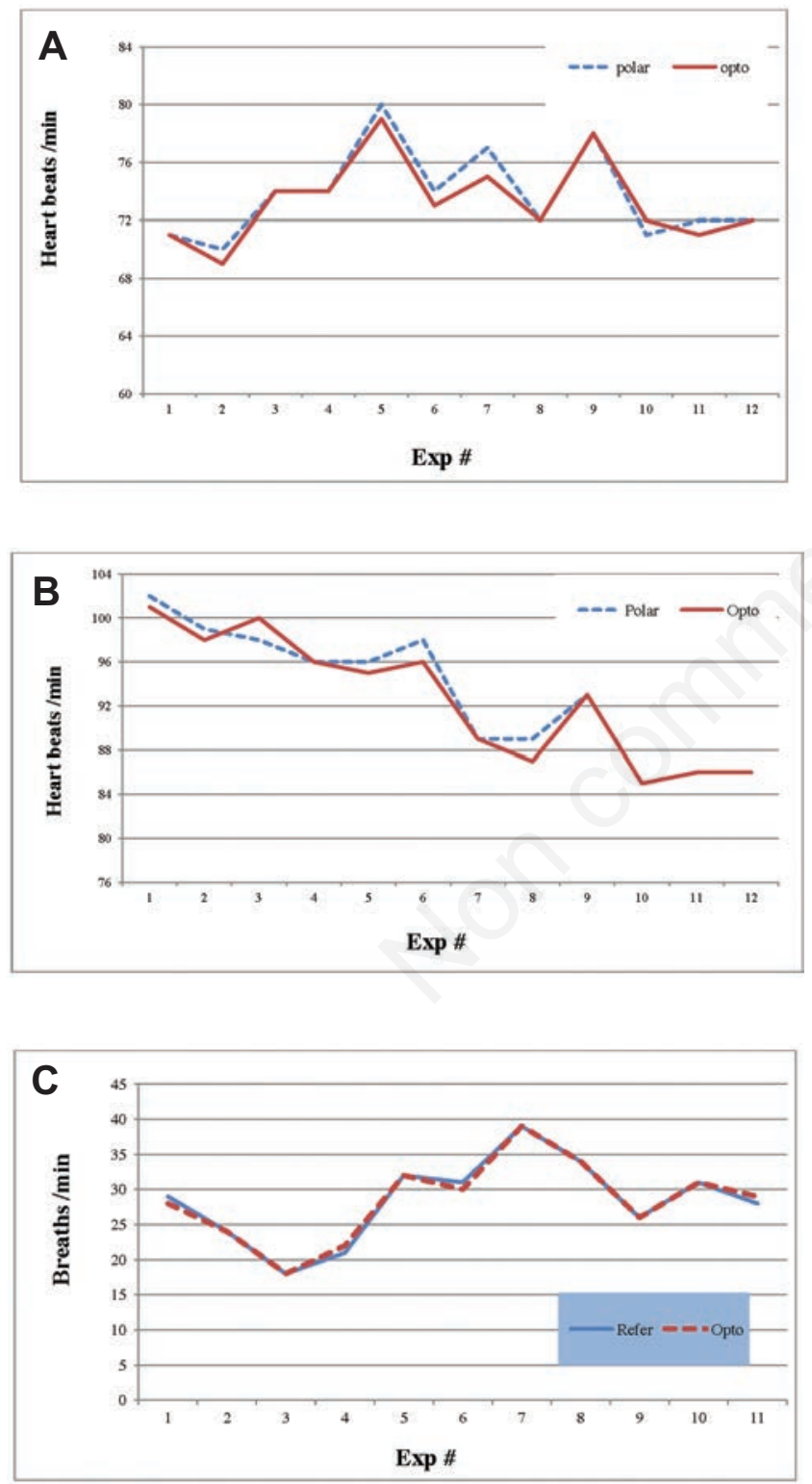

Figure 7. Cows' biomedical measurement collection. The blue dashed line denotes to the reference measurement, while the red solid line to the optical readout. A) the first cow heart beats; B) the second cow heart beats; C) breathing rate by a third cow. for every amount of peaks. One can notice that the optimal threshold is indeed different for different peak numbers.

Figure 10 presents the best six threshold values for any amount of Gaussian curves.

Selecting the best threshold requires additional computation since it involves testing of the probability map. We examined to what extent wrong selection of threshold value will reduce the performances as expressed through efficiency. Figure 11 presents the performances of the best and worst threshold value which are also compared to the greedy probabilistic algorithm (Shenhav et al., 2012). It is clear that that the best threshold has a clear advantage. However, the efficiency of the worst threshold value is still good and way beyond the greedy probabilistic algorithm.

\section{Cow number identification}

The algorithm efficiency is presented in Table 1. Different daylight conditions were examined, from bright sunlight illumination to foggy winter halfdarkness. Very frequently the freeze brand zone has been in a shadow and the contrast of characters has been poor with regard to the freeze brand's background. Situations of mixed illumination, where certain portions of the freeze brand were shadowed, while the others were brightly illuminated, caused problems and sometimes led to rejection of the whole freeze brand.

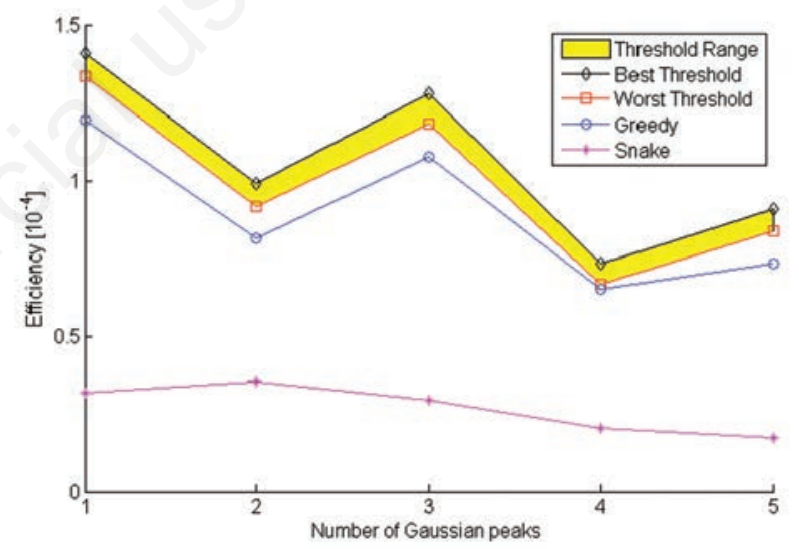

Figure 8. Comparison of efficiency of several algorithms.

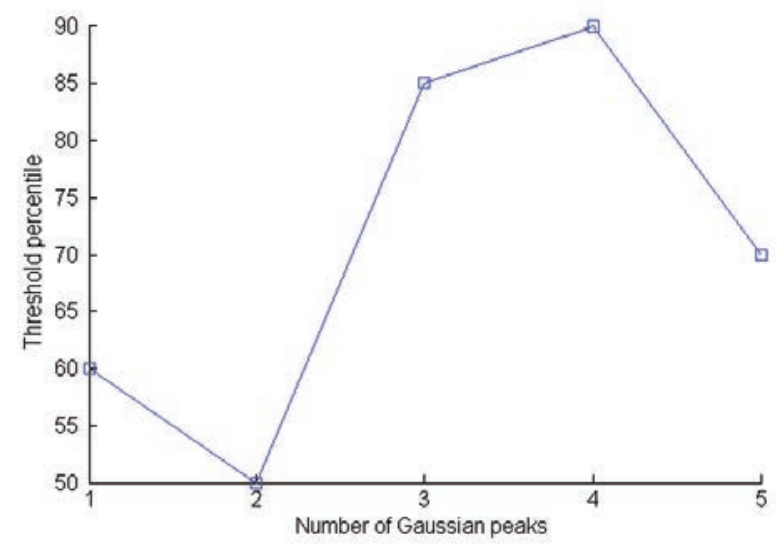

Figure 9. Change of threshold value according to peak numbers. 

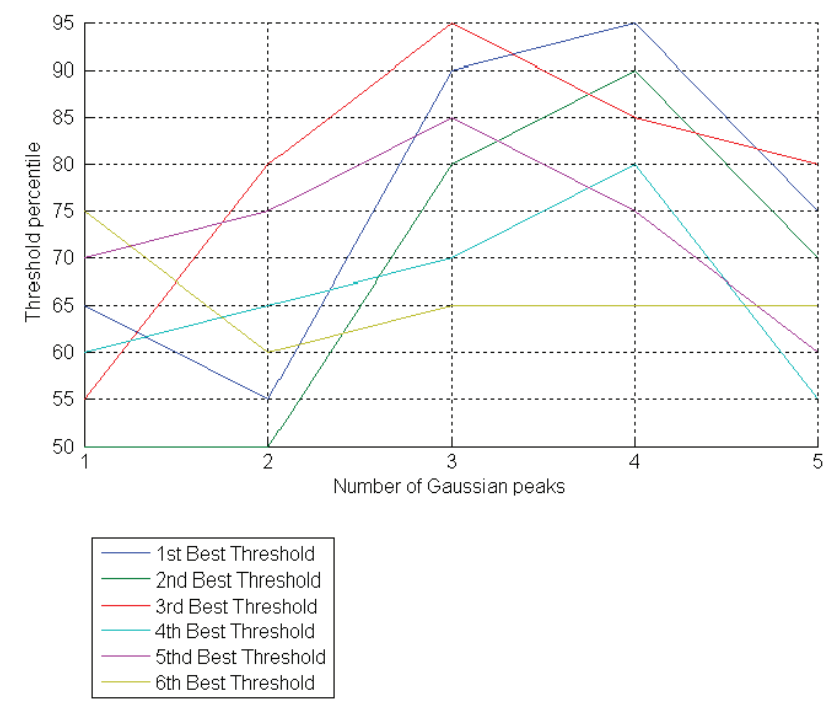

Figure 10. Change of threshold value according to peak numbers.

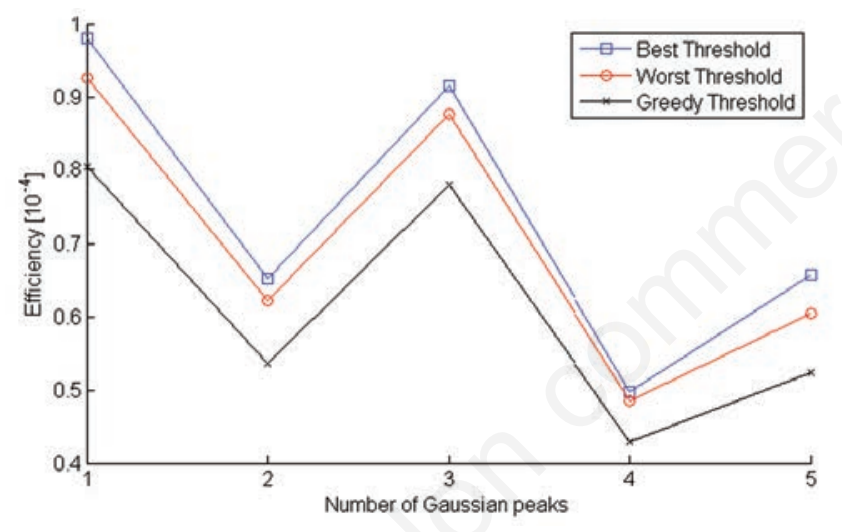

Figure 11. Comparison of threshold value efficiencies.

Table 1. Chosen algorithm efficiency.

\begin{tabular}{lccc} 
& $\begin{array}{c}\text { No. } \\
\text { of brands }\end{array}$ & $\begin{array}{c}\text { No. } \\
\text { of characters }\end{array}$ & $\begin{array}{c}\text { Success } \\
(\%)\end{array}$ \\
Clear brand & 68 & 204 & 87.2 \\
Blurred brand & 52 & 156 & 46.87 \\
\hline Skewed brand & 40 & 120 & 51.64 \\
Total & 160 & 480 & - \\
\hline Average & - & - & 65.20 \\
\hline
\end{tabular}

\section{Discussion}

Among advantages of the proposed system one can stress that system is comprised only one sensor, while is operability covers three different tasks: identification, detection and biomedical monitoring. The biomedical monitoring is not dependant on the illumination of the subject, does not require high image signal-to-noise ratio (can operate at night). The image processing techniques are robust and show a reliable outcome by the simulations. Only few images of extremely poor quality (poor contrast or missing part) attempted more than three prospective strips. The conclusion is that in case of reasonably good images the above-described freeze brand localisation approach yields good results. The system is low cost as it is uses off-the-shelf components and easy to use, no additional hardware such as transmitters mounted on the cow or additional sensors are required.

Some disadvantages can be named here: high air humidity cause bluer on lens camera that makes hardly cow identification. In addition, the system is still in the development stage; therefore it is not fully explored with the real-time cases.

\section{Conclusions}

The field tests with cows have shown a very good feasibility for monitoring all three of the chosen biomedical parameters: pulse count (heart beat rate), breathing activity, and chewing. The accuracy of the measurement was as good as the sampling time interval (bit to bit counting), while the reference measurements matched our recordings.

A system that integrates the optical sensor with a digital camera can be placed on a moving robotic system above a cowshed area. When the optical sensor identifies a cow, the system stops, collects the cow biomedical data and number and transmits it to a host computer. The proposed algorithms were developed and its simulation outcome presented a good potential for an automatic cows monitoring.

\section{References}

Alhanati V., Halahmi I., Cohen Y., Antler A., Greenshpan Y., Ostrovsky V., Palmenbaum I., Shushani E. 2010. Optimal operation of cows cooling system based on automatic crowding detection and air flow. AR0, Israel, research \# 458-0331-04(Hebrew).

Battaglia R.A. 2001. Handbook of livestock management. $3^{\text {rd }}$ ed. Prentice-Hall Inc, Englewood Cliffs, NJ, USA.

Beiderman Y., Amsel A.D., Tzadka Y., Fixler D., Mico V., Garcia J., Zalevsky Z. 2011a. A microscope configuration for nanometer 3-D movement monitoring accuracy. Micron 42:366-75.

Beiderman Y., Blumenberg R., Rabani N., Teicher M., Garcia J., Mico V., Zalevsky Z. 2011b. Optical sensor for remote estimation of glucose concentration in blood. Biomed. Opt. Exp. 2:858-70.

Beiderman Y., Horovitz I., Burshtein N., M. Teicher M., Garcia J., Mico V., Zalevsky Z. 2010. Remote estimation of blood pulse pressure via temporal tracking of reflected secondary speckles pattern. J. Biomed. 0pt. 15:061707-1-7.

Beiderman Y., Talyosef R., Yeori D., J. Garcia J., Mico V., Zalevsky Z. 2012. The usage of PC mouse components for continuous measuring of human heart beats. Appl. 0pt. 51:3323-8.

Brosh A. 2007. Heart rate measurements as an index of energy expenditure and energy balance in ruminants: a review. J. Anim. Sci. $85: 1213-27$. 
Brosh A., Henkin Z., Ungar E.D., Dolev A., Orlov A., Yehuda Y., Aharoni Y. 2006. Energy cost of cows' grazing activity: Use of the heart rate method and the global positioning system for direct field estimation. J. Anim. Sci. 84:1951-67.

Chu T.C., Ranson W.F., Sutton M.A. 1985. Applications of digital-imagecorrelation techniques to experimental mechanics. Exp. Mech 25:232-44.

Cojoc D., Finaurini S., Livshits P., Gur E., Shapira A., Mico V., Zalevsky Z. 2012. Early detection of malaria by non contact photonic means. Biomed. Opt. Exp. 3:991-1005.

Dainty J.C. 1989. Laser speckle and related phenomena, 2nd ed. Springer-Verlag, Berlin, Germany.

Garcia J., Zalevsky Z., Fixler D. 2005. Synthetic aperture superresolution by speckle pattern projection. Opt. Exp. 13:6073-6078.

García J., Zalevsky Z., García-Martínez P., Ferreira C., Teicher M., Beiderman Y., Shpunt A. 2008. 3D mapping and range measurement by means of projected speckle patterns. Appl. Opt. 47:3032-40.

Halachmi I., Polak P., Roberts D., Klopcic M. 2008. Cow body shape and automation of condition scoring BCS. J. Dairy Sci. 91:4444-51.

Kim J., Young Jung W. 2013. Vision-based cattle detection and localization system in an RGB color space. INTELLI 2013: 2nd Int. Conf. on Intelligent Systems and Applications, April 21-26, Venice, Italy

Leedertz J.A. 1970. Interferometric displacement measurements on scattering surfaces utilizing speckle effects. J. Phy. E. Sci. Instrum. 3:214-8.

Margalit I., Beiderman Y., Skaat A., Rosenfeld E., Belkin M., Tornow R.P., Mico V., Garcia J., Zalevsky Z. 2014. A new method for remote continuous monitoring of intraocular pressure variations. J. Biomed. Opt. 19:027002.
Pedersen H.M. 1982. Intensity correlation metrology: a comparative study. Opt. Acta 29:105-18.

Pelikan M., Goldberg D.E., F. Lobo F. 2002. A survey of optimization by building and using probabilistic models. Comp. Opt. App. 21:5-20.

Peters W.H., Ranson W.F. 1982. Digital imaging techniques in experimental stress analysis. 0pt. Eng. 21:427-31.

Rastogi P.K., Jacquot P. 1987. Measurement on difference deformation using speckle interferometry. Opt. Lett. 12:596-8.

Shapiro V., Dimov D., Bonchev S., Velichkov V., Gluhchev G. 2004. Adaptive license plate image extraction. Proc. Int. Conf. Computer Systems and Technologies, Rousse, Bulgaria.

Shenhav A., Brodie Z., Beiderman Y., Garcia J., Mico V., Zalevsky Z. 2012. Optical sensor for remote estimation of alcohol concentration in blood. Opt. Commun. 289:149-57.

Stankovsky S., Ostojic G., Senk I., Rakic-Skokovic M., Trivunovic S., Kucevic D. 2012. Dairy cow monitoring by RFID. Sci. Agric. 69:75-80.

Takai N., Iwai T., Ushizaka T., Asakura T. 1980. Zero crossing study on dynamic properties of speckles. J. Opt. (Paris) 11:93-101.

Uno K., Uozumi J., Asakura T. 1996. Correlation properties of speckles produced by diffractal-illuminated diffusers. Opt. Commun. 124:16-22.

Zalevsky Z., García J. 2007. Motion detection system and method. Israeli Patent Application No. 184868 (July 2007); WO/2009/013738 International Application No. PCT/IL2008/001008 (July 2008).

Zalevsky Z., Beiderman Y., Margalit I., Gingold S., Teicher M., Mico V., Garcia J. 2009. Simultaneous remote extraction of multiple speech sources and heart beats from secondary speckles pattern. Opt. Exp. 17:21566-80. 